\title{
Preoperative statin therapy reduces postoperative all-cause mortality in cardiac surgery: A meta-analysis of controlled studies
}

\author{
Hisato Takagi, MD, PhD, Norikazu Kawai, MD, and Takuya Umemoto, MD, PhD, Shizuoka, Japan
}

Two recent meta-analyses ${ }^{1,2}$ demonstrated that postoperative mortality was significantly lower in patients undergoing cardiac surgery who received preoperative statin therapy than in those who did not. The meta-analysis by Kapoor and associates, ${ }^{1}$ however, included merely 4 published articles, and that by Hindler and colleagues ${ }^{2}$ cited 4 published articles and 3 scientific abstracts. Therefore, the appropriate role of preoperative statin therapy in cardiac surgery remains unclear. Furthermore, several controlled studies of preoperative statin therapy in cardiac surgery have been published to date since the 2 meta-analyses were conducted. Herein, we performed a meta-analysis of controlled studies of preoperative statin therapy for prevention of postoperative all-cause mortality in cardiac surgery.

\section{MATERIALS AND METHODS}

All controlled studies (English-language full-text journal publications excluding scientific abstracts) of preoperative statin therapy in cardiac surgery were identified by a 2-level search strategy. First, a public domain database (MEDLINE) was searched using a Web-based search engine (PubMed). Second, relevant studies were identified through a manual search of secondary sources including references of initially identified articles and a search of reviews and commentaries. The MEDLINE database was searched from January 1966 to December 2007. MeSH keywords included "Hydroxymethylglutaryl-CoA Reductase Inhibitors" and "Cardiac Surgical Procedures." Studies considered for inclusion met the following criteria: the design was a controlled study of preoperative statin therapy; the study population was patients undergoing cardiac surgery; and main outcomes included postoperative all-cause mortality. Data regarding detailed inclusion criteria and mortality were abstracted (as available) from each individual study. For each study, data regarding mortality in both the statin and control (placebo or no statin) groups were used to generate crude odds ratios (ORs) and $95 \%$ confidence intervals (CIs). We also abstracted adjusted ORs and $95 \%$ CIs for mortality if reported. Study-specific estimates were combined using inverse variance-weighted averages of logarithmic ORs in a randomeffects model. Between-study heterogeneity was analyzed by means of standard $\chi^{2}$ tests. Publication bias was assessed mathematically using an adjusted rank-correlation test.

\section{RESULTS}

Our search identified 13 controlled studies of preoperative statin therapy in cardiac surgery, the majority of

From the Department of Cardiovascular Surgery, Shizuoka Medical Center, Shizuoka, Japan.

Received for publication Jan 22, 2008; accepted for publication Feb 24, 2008.

Address for reprints: Hisato Takagi, MD, PhD, Department of Cardiovascular Surgery, Shizuoka Medical Center, 762-1 Nagasawa, Shimizu-cho, Sunto-gun, Shizuoka 411-8611, Japan (E-mail: kfgth973@ybb.ne.jp).

J Thorac Cardiovasc Surg 2009;137:e52-3

$0022-5223 / \$ 36.00$

Copyright (c) 2009 by The American Association for Thoracic Surgery doi: $10.1016 /$ j.jtcvs.2008.02.045 which was coronary artery bypass graft surgery (Table 1). These included 3 randomized controlled trials, ${ }^{3-5} 3$ prospective cohort studies, and 7 retrospective cohort studies. Pooled analysis of crude ORs from all 13 studies (representing 19,542 patients) demonstrated a statistically significant $45 \%$ reduction in postoperative all-cause mortality with preoperative statin therapy relative to control (pooled crude OR, 0.55; 95\% CI, 0.46-0.66; $P<.01$ ). There was neither study heterogeneity of results $(P=$ .64) nor evidence of significant publication bias $(P=$ .74). Six of the 10 observational studies reported adjusted ORs for mortality by multivariate analysis or propensity score matching. When adjusted ORs from these 6 observational studies and ORs from the 3 randomized controlled trials ${ }^{3-5}$ were pooled (representing 18,637 patients), preoperative statin therapy was associated with a $25 \%$ reduction in mortality relative to control that remained statistically significant (pooled adjusted OR, 0.76; 95\% CI, 0.64-0.90; $P<.01)$. There was neither study heterogeneity of results $(P=.60)$ nor evidence of significant publication bias $(P=.53)$. Pooled analysis of ORs and adjusted Ors, respectively, from 2 randomized controlled trials ${ }^{3,4}$ and 3 observational studies that enrolled patients undergoing coronary artery bypass graft surgery exclusively (representing 7205 patients) demonstrated a statistically significant $41 \%$ reduction in mortality with preoperative statin therapy relative to control (adjusted OR, 0.59; 95\% CI, 0.36-0.97; $P=.04)$. There was neither study heterogeneity of results $(P=.57)$ nor evidence of significant publication bias $(P=.62)$.

\section{DISCUSSION}

The present meta-analysis of 13 controlled studies demonstrated that preoperative statin therapy reduced postoperative all-cause mortality in cardiac surgery. The analysis, however, included 10 observational studies but merely 3 small randomized controlled trials (sample size: 40, 77, and 200). ${ }^{3-5}$ In an attempt to correct for and minimize selection bias, which exists in observational studies, related to statin use, we pooled adjusted instead of crude ORs from the observational studies and ORs from the randomized controlled trials, resulting in a statistically significant benefit of preoperative statin therapy for mortality in cardiac surgery or exclusive coronary artery bypass graft surgery. Nevertheless, larger randomized controlled trials are needed to confirm our results. Until sufficient numbers of events will be accumulated by evidence from randomized controlled trials to provide a definitive answer, 
TABLE 1. Characteristics and outcomes of included studies

\begin{tabular}{|c|c|c|c|c|c|c|}
\hline \multirow[b]{2}{*}{ Author } & \multirow[b]{2}{*}{ Publication } & \multirow[b]{2}{*}{ Design } & \multirow[b]{2}{*}{ Sample size } & \multirow[b]{2}{*}{ Type of operation } & \multicolumn{2}{|c|}{ OR $(95 \%$ CI $)$ for mortality } \\
\hline & & & & & Crude & Adjusted \\
\hline Ali and Buth & $\begin{array}{l}\text { Int J Cardiol. } \\
\qquad 2005 ; 103: 12-8\end{array}$ & Retrospective cohort & 5,469 & $\begin{array}{l}\text { Cardiac surgery (Isolated } \\
\text { CABG } 80 \% \text { ) }\end{array}$ & $0.50(0.38-0.67)$ & $0.87(0.61-1.25)^{*}$ \\
\hline Chello et $\mathrm{al}^{4}$ & $\begin{array}{l}\text { Crit Care Med. } \\
\text { 2006;34:660-7 }\end{array}$ & Randomized controlled & 40 & Elective isolated CABG & $1.00(0.02-52.85)$ & \\
\hline Christenson $^{3}$ & $\begin{array}{l}\text { Eur J Cardiothorac Surg. } \\
\text { 1999;15:394-400 }\end{array}$ & Randomized controlled & 77 & Elective isolated CABG & $0.93(0.02-47.85)$ & \\
\hline Clark et al & $\begin{array}{l}\text { J Thorac Cardiovasc } \\
\quad \text { Surg. 2006;131: } \\
\quad 679-85\end{array}$ & Retrospective cohort & 3,829 & $\begin{array}{l}\text { Elective cardiac surgery } \\
\quad(\text { any CABG } 81 \% \text { ) }\end{array}$ & $0.43(0.28-0.66)$ & $0.51(0.27-0.94)^{*}$ \\
\hline Coleman et al & $\begin{array}{l}\text { Curr Med Res Opin. } \\
\text { 2007;23:1783-90. }\end{array}$ & Retrospective cohort & 1,934 & $\begin{array}{l}\text { Cardiac surgery (isolated } \\
\text { CABG } 63 \% \text { ) }\end{array}$ & $0.68(0.45-1.03)$ & $0.80(0.64-0.99) \dagger$ \\
\hline Collard et al & $\begin{array}{l}\text { J Thorac Cardiovasc } \\
\text { Surg. 2006;132: } \\
\text { 392-400 }\end{array}$ & Prospective cohort & 2,666 & $\begin{array}{l}\text { Elective primary isolated } \\
\text { CABG }\end{array}$ & $0.79(0.47-1.33)$ & $0.04(0.08-0.93) \dagger$ \\
\hline Dotani et al & $\begin{array}{l}\text { Am J Cardiol. } \\
\quad \text { 2000;86:1128-30, A6 }\end{array}$ & Retrospective cohort & 323 & Elective isolated CABG & $0.12(0.01-2.08)$ & NR \\
\hline Liakopoulos et al & $\begin{array}{l}\text { Thorac Cardiovasc Surg. } \\
2006 ; 54: 250-4\end{array}$ & Prospective cohort & 36 & Elective isolated CABG & $1.00(0.02-53.12)$ & NR \\
\hline Mariscalco et al & $\begin{array}{l}\text { Ann Thorac Surg. } \\
\text { 2007;84:1158-64 }\end{array}$ & Retrospective cohort & 405 & $\begin{array}{l}\text { Elective primary isolated } \\
\text { CABG }\end{array}$ & $0.28(0.01-7.03)$ & NR \\
\hline Pan et al & $\begin{array}{l}\text { Circulation. 2004;110(11 } \\
\text { Suppl 1):II45-9 }\end{array}$ & Retrospective cohort & 1,663 & Primary isolated CABG & $0.47(0.25-0.87)$ & $0.58(0.31-1.09)^{*}$ \\
\hline Pascual et al & $\begin{array}{c}\text { Ann Thorac Surg. } \\
2006 ; 81: 78-83\end{array}$ & Prospective cohort & 141 & Elective isolated CABG & $0.16(0.03-0.79)$ & NR \\
\hline Patti et $\mathrm{al}^{5}$ & $\begin{array}{l}\text { Circulation. } \\
\text { 2006;114:1455-61 }\end{array}$ & Randomized controlled & 200 & $\begin{array}{l}\text { Elective cardiac surgery } \\
\quad \text { (isolated CABG 79\%) }\end{array}$ & $0.98(0.14-7.10)$ & \\
\hline Thielmann et al & $\begin{array}{l}\text { J Thorac Cardiovasc } \\
\text { Surg. 2007;134: } \\
1143-9\end{array}$ & Retrospective cohort & 2,759 & $\begin{array}{l}\text { Elective primary isolated } \\
\text { CABG }\end{array}$ & $1.08(0.39-3.00)$ & $1.18(0.36-3.87)^{*}$ \\
\hline Total & & & 19,542 & & $0.55(0.46-0.66) \ddagger$ & $0.76(0.64-0.90) \ddagger$ \\
\hline
\end{tabular}

advocating routine preoperative statin therapy in cardiac surgery is premature.

\section{References}

1. Kapoor AS, Kanji H, Buckingham J, Devereaux PJ, McAlister FA. Strength of evidence for perioperative use of statins to reduce cardiovascular risk: systematic review of controlled studies. BMJ. 2006;333:1149.

2. Hindler K, Shaw AD, Samuels J, Fulton S, Collard CD, Riedel B. Improved postoperative outcomes associated with preoperative statin therapy. Anesthesiology. 2006;105:1260-72.
3. Christenson JT. Preoperative lipid-control with simvastatin reduces the risk of postoperative thrombocytosis and thrombotic complications following CABG. Eur J Cardiothorac Surg. 1999;15:394-400.

4. Chello M, Patti G, Candura D, Mastrobuoni S, Di Sciascio G, Agrò F, et al. Effects of atorvastatin on systemic inflammatory response after coronary bypass surgery. Crit Care Med. 2006;34:660-7.

5. Patti G, Chello M, Candura D, Pasceri V, D'Ambrosio A, Covino E, et al. Randomized trial of atorvastatin for reduction of postoperative atrial fibrillation in patients undergoing cardiac surgery: results of the ARMYDA-3 (Atorvastatin for Reduction of MYocardial Dysrhythmia After cardiac surgery) study. Circulation. 2006;114:1455-61. 